\title{
A robust SARS-CoV-2 replication model in primary human epithelial cells at the air liquid interface to assess antiviral agents
}

\author{
Thuc Nguyen Dan Do ${ }^{\mathrm{a}}$, Kim Donckers ${ }^{\mathrm{a}}$, Laura Vangeel ${ }^{\mathrm{a}}$, Arnab K. Chatterjee ${ }^{\mathrm{b}}$, \\ Philippe A. Gallay ${ }^{c}$, Michael D. Bobardt ${ }^{c}$, John P. Bilello ${ }^{\mathrm{d}}$, Tomas Cihlar ${ }^{\mathrm{d}}$, Steven De Jonghe ${ }^{\mathrm{a}}$, \\ Johan Neyts $^{\text {a, }}$, Dirk Jochmans ${ }^{\text {a, *** }}$ \\ ${ }^{\text {a }}$ KU Leuven - Department of Microbiology, Immunology and Transplantation, Rega Institute, Laboratory of Virology and Chemotherapy, Leuven, Belgium \\ ${ }^{\mathrm{b}}$ CALIBR - Department of Medicinal Chemistry, The Scripps Research Institute, La Jolla, CA, USA \\ ${ }^{\mathrm{c}}$ CALIBR - Department of Immunology and Microbial Science, The Scripps Research Institute, La Jolla, CA, USA \\ d Gilead Sciences, Inc., Foster City, CA, USA
}

\section{A R T I C L E I N F O}

\section{Keywords:}

SARS-CoV-2

Antivirals

Primary human airway epithelial cells

Remdesivir

GS-441524

EIDD-1931

\begin{abstract}
A B S T R A C T
There are, besides remdesivir, no approved antivirals for the treatment of SARS-CoV-2 infections. To aid in the search for antivirals against this virus, we explored the use of human tracheal airway epithelial cells (HtAEC) and human small airway epithelial cells (HsAEC) grown at the air-liquid interface (ALI). These cultures were infected at the apical side with one of two different SARS-CoV-2 isolates. Each virus was shown to replicate to high titers for extended periods of time (at least 8 days) and, in particular an isolate with the D614G in the spike (S) protein did so more efficiently at $35^{\circ} \mathrm{C}$ than $37^{\circ} \mathrm{C}$. The effect of a selected panel of reference drugs that were added to the culture medium at the basolateral side of the system was explored. Remdesivir, GS-441524 (the parent nucleoside of remdesivir), EIDD-1931 (the parent nucleoside of molnupiravir) and IFN ( $\beta 1$ and $\lambda 1$ ) all resulted in dose-dependent inhibition of viral RNA and infectious virus titers collected at the apical side. However, AT-511 (the free base form of AT-527 currently in clinical testing) failed to inhibit viral replication in these in vitro primary cell models. Together, these results provide a reference for further studies aimed at selecting SARS-CoV2 inhibitors for further preclinical and clinical development.
\end{abstract}

\section{Introduction}

Remdesivir is currently the only approved antiviral for the treatment of COVID-19 (Beigel et al., 2020). Major efforts are ongoing to develop novel antiviral drugs for effective COVID-19 treatment. To aid in their development, physiologically-relevant in vitro models are needed. Immortalized cell lines originating from non-respiratory (and often non-human) tissues are frequently used in early preclinical studies for antiviral assessment. For example, VeroE6, a widely used cell line in SARS-CoV-2 studies, is defective in the expression of main SARS-CoV-2 receptors (angiotensin-converting enzyme 2 (ACE2) and transmembrane protease serine 2 (TMPRSS2)). Hence, screening campaigns often result in the discovery of antiviral agents that regulate autophagy pathways and endosomal-lysosomal maturation, which may not be pertinent or translatable as SARS-CoV-2 therapies (Murgolo et al., 2021).
Meanwhile, air-liquid interface (ALI) of differentiated primary human airway epithelial cells (HAEC) possess the architecture and cellular complexity of human lung tissue and are permissive to variety of respiratory viral infections (Boda et al., 2018; Loo et al., 2020). Containing all relevant cell types of the lower respiratory tract (ciliated, goblet and basal cells), which includes ACE2 and TMPRSS2 expressing cells, this system allows dissection of the host-pathogen interactions at molecular and cellular levels and provides a platform for profiling antiviral drugs.

In this study, we explored the effects of several reported SARS-CoV-2 inhibitors on the replication of different SARS-CoV-2 isolates in HAEC ALI cultures focusing the testing primarily on the nucleoside class of inhibitors with previously reported in vitro anti-SARS-CoV-2 activity. Our results provide a reference set of data for these relevant in vitro cell models to support the preclinical development of SARS-CoV-2 inhibitors.

\footnotetext{
* Corresponding author.

$* *$ Corresponding author.

E-mail addresses: johan.neyts@kuleuven.be (J. Neyts), dirk.jochmans@kuleuven.be (D. Jochmans).
} 


\section{Materials and methods}

\subsection{Cells and virus isolates}

The African monkey kidney cell line VeroE6 tagged green fluorescent protein (VeroE6-GFP, kindly provided by M. van Loock, Janssen Pharmaceutica, Beerse, Belgium) and VeroE6 were maintained in Dulbecco's modified Eagle's medium (DMEM; Gibco, catalogue no. 41965-039) supplemented with $10 \% \mathrm{v} / \mathrm{v}$ heat-inactivated foetal bovine serum (HIFBS; HyClone, catalogue no. SV03160.03), 1\% v/v sodium bicarbonate $7.5 \% \mathrm{w} / \mathrm{v}\left(\mathrm{NaHCO}_{3}\right.$; Gibco, catalogue no. 25080-060), and 1\% v/v Penicillin-Streptomycin $10000 \mathrm{U} / \mathrm{mL}$ (P/S; Gibco, catalogue no. 15140148 ) at $37{ }^{\circ} \mathrm{C}$ and $5 \% \mathrm{CO}_{2}$. The hepatocellular carcinoma cell line Huh7 (kindly provided by Ralf Bartenschlager, University of Heidelberg, Germany) was propagated in DMEM supplemented with $10 \%$ HI-FBS, $1 \% \mathrm{NaHCO}_{3}, 1 \% \mathrm{P} / \mathrm{S}, 1 \%$ non-essential amino acids (NEAA; Gibco, catalogue no. 11140050), and 2\% HEPES 1M (Gibco, catalogue no. 15630106) at $37{ }^{\circ} \mathrm{C}$ and $5 \% \mathrm{CO}_{2}$. All assays involving virus growth were performed in the respective cell growth medium containing $2 \%$ (VeroE6-GFP) or 4\% (Huh7) instead of 10\% FBS.

SARS-CoV-2 isolate BetaCoV/Germany/BavPat1/2020 (EPIISL_406862|2020-01-28, kindly provided by C. Drosten, Charité, Berlin, Germany) and BetaCov/Belgium/GHB-03021/2020 (EPI_ISL_407976| 2020-02-03) retrieved from RT-qPCR-confirmed COVID-19 positive patients in January and February 2020 were described previously (Bohmer et al., 2020; Dellicour et al., 2021). The generation of virus stocks by serial passaging in Huh7 and VeroE6 cells were fully reported (Boudewijns et al., 2020; Rut et al., 2021). BavPat1 isolate (passage 2 (P2)) and GHB-03021 isolate (P6 and P7) were used for the air liquid-interface experiment while only the latter was used for standard in vitro assays in VeroE6-GFP cells (P6 and P7) and in Huh7 cells (P9). The genomic sequence of both isolates is highly similar. BavPat 1 carries the D614G amino acid change in the spike-protein while the GHB-03021 has a $\triangle$ TQTNS deletion at $676-680$ residues that is typical for SARS-CoV-2 strains that have been passaged several times on VeroE6 cells. All infectious virus-containing works were conducted in biosafety level 3 (BSL-3) and 3+ (CAPs-IT) facilities at the Rega Institute for Medical Research, KU Leuven, according to institutional guidelines.

\subsection{Compounds}

Remdesivir was synthesized at Gilead Sciences, Inc. (Foster City, CA). GS-441524 was either synthesized at Gilead (Fig. 2) or it was purchased from Carbosynth (United Kingdom) (other figures). EIDD1931 was purchased from R\&D Systems (USA). Stock solutions (10 $\mathrm{mM}$ ) were prepared using analytical grade dimethyl sulfoxide (DMSO). AT-511 was synthesized and chemically validated at the California Institute for Biochemical Research (Calibr) (La Jolla, CA) and used as a $10 \mathrm{mM}$ DMSO solution. The biological activity of AT-511 was confirmed in an antiviral assay with hepatitis $C$ virus (data not shown). IFN $\lambda 1$ was purchased from R\&D Systems and IFN $\beta$-1a was a kind gift from the laboratory of Immunobiology (Rega Institute, KU Leuven, Belgium). Both were reconstituted in sterile phosphate buffered saline (PBS, Life Technologies) containing at least $0.1 \%$ FBS.

\subsection{In vitro standard antiviral and toxicity assays}

VeroE6-GFP cells were seeded at a density of 25000 cells/well in 96well plates (Greiner Bio One, catalogue no. 655090) and pre-treated with three-fold serial dilutions of the compounds overnight. On the next day (day 0), cells were infected with the SARS-CoV-2 inoculum at a multiplicity of infection (MOI) of 0.001 median tissue culture infectious dose (TCID50) per cell. The number of fluorescent pixels of GFP signal determined by High-Content Imaging (HCI) on day 4 post-infection (p. i.) was used as a read-out. Percentage of inhibition was calculated by subtracting background (number of fluorescent pixels in the untreatedinfected control wells) and normalizing to the untreated-uninfected control wells (also background subtracted). The 50\% effective concentration $\left(\mathrm{EC}_{50}\right.$, the concentration of compound required for fifty percent recovery of cell-induced fluorescence) was determined using logarithmic interpolation. Potential toxicity of compounds was assessed in a similar set-up in treated-uninfected cultures where metabolic activity was quantified at day 5 using the MTS assay as described earlier (Jochmans et al., 2012). The 50\% cytotoxic concentration $\left(\mathrm{CC}_{50}\right.$, the concentration at which cell viability reduced to $50 \%$ ) was calculated by logarithmic interpolation.

Huh7 cells were pre-seeded at 6000 cells/well in 96 well-plates (Corning, catalogue no.3300) and incubated overnight at $37{ }^{\circ} \mathrm{C}$ and $5 \% \mathrm{CO}_{2}$. On day 0 , cells were firstly treated with the three-fold serial dilution of a potential antiviral, followed by either the inoculation of
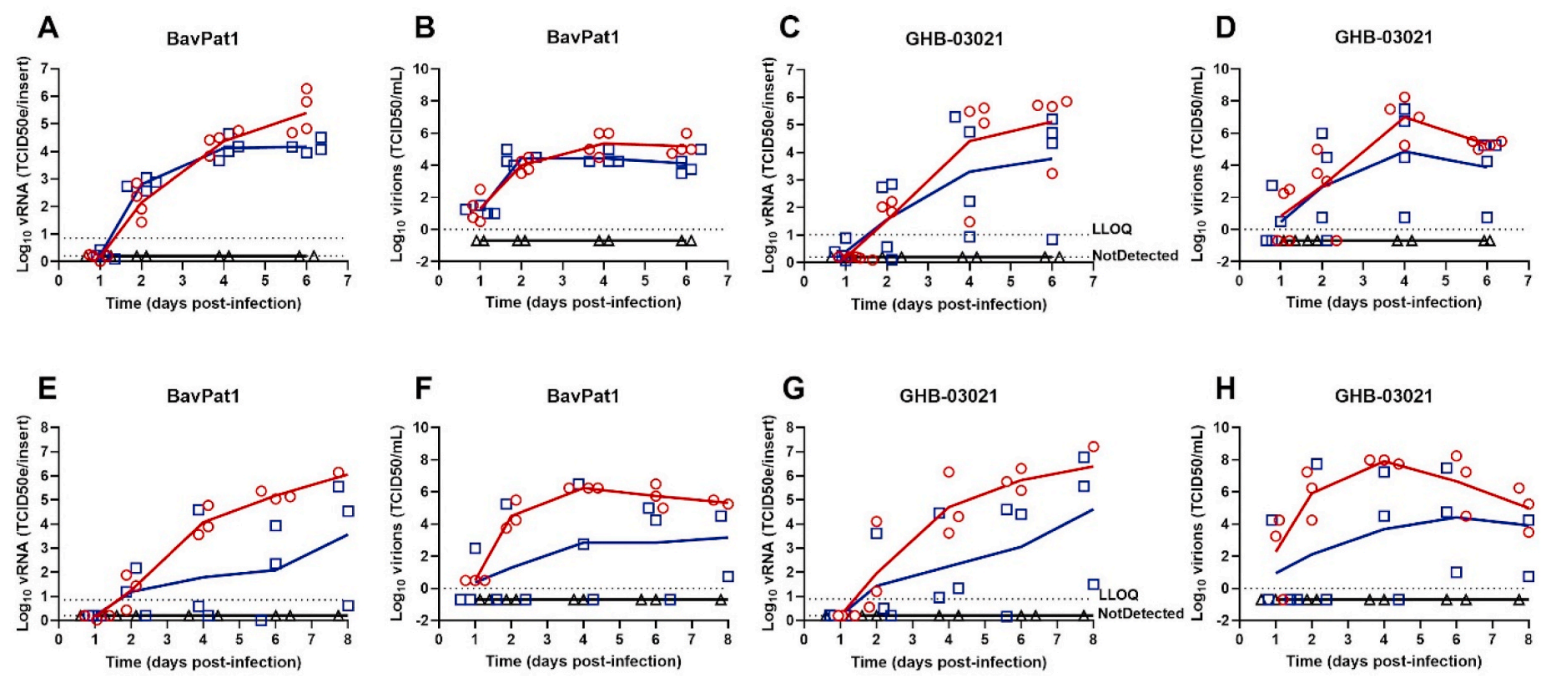

Fig. 1. SARS-CoV-2 replication kinetics in air-liquid interface cultures. Viral replication of BavPat1 and GHB-03021 isolates at $2 \times 10^{3}$ TCID50/insert in human tracheal airway epithelia cells (A-D) or in human small airway epithelia cells (E-H) at either $35^{\circ} \mathrm{C}$ (red circle) or $37^{\circ} \mathrm{C}$ (blue square) in comparison with uninfected control (black triangle). Viral RNA or infectious particles in apical washes were quantified by RT-qPCR (A, C, E, G) or by end-point titrations (B, D, F, H), respectively. The results of individual inserts are depicted as dots. The line follows the average of each conditions. 

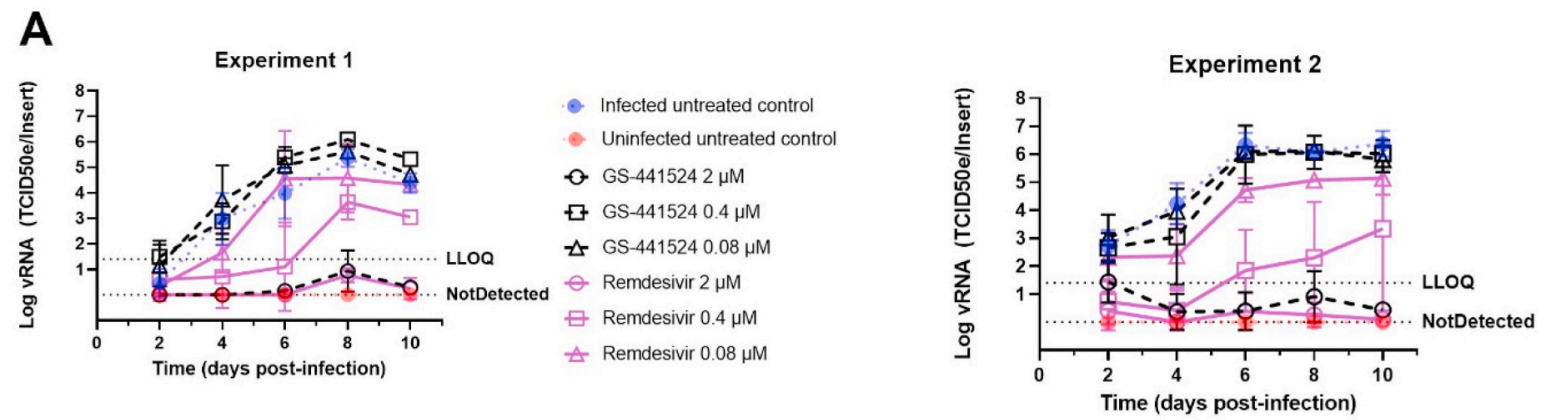

\section{B}
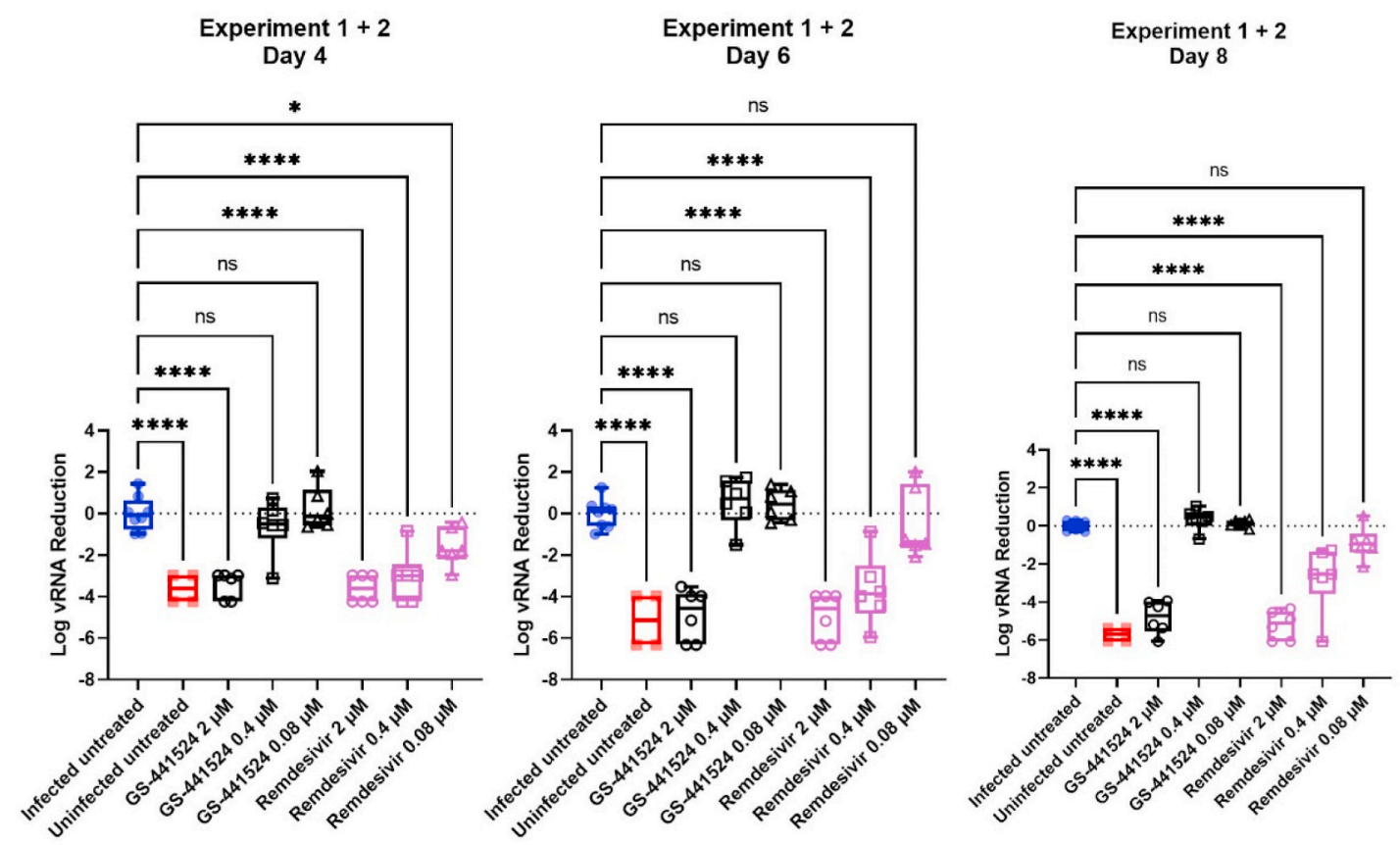

Fig. 2. Remdesivir and GS-441524 inhibit SARS-CoV-2 replication in human tracheal airway cultures. Compounds were added to the basal medium starting 2 $\mathrm{h}$ before infection and treatment continued for 10 days. HtAEC cultures were infected with SARS-CoV- 2 GHB-03021 at $2 \times 10^{4}$ TCID50/insert and incubated at $37^{\circ} \mathrm{C}$. Viral RNA in apical washes was quantified by RT-qPCR. (A) Kinetics of viral replication. Two independent experiments with HtAEC from different donors were performed. In each experiment 3 independent inserts were used for each compound concentration. LLOQ represent the lower limit of quantification. (B) The Log vRNA Reduction on day 4, 6 and 8 of the experiment. For this analysis the samples of both experiments were pooled. The Log vRNA Reduction was calculated by subtracting the average Log vRNA of the infected untreated samples for that particular day from the Log vRNA of each sample. ns $p>0.05$, *p $<0.05$, ${ }^{* *} \mathrm{p}<0.01$, $* * * \mathrm{p}<0.001, * * * * \mathrm{p}<0.0001$

SARS-CoV-2 at MOI of 0.0037 TCID50/cell or addition of fresh medium. After 4 days, differences in cell viability caused by virus-induced cytopathic effect (CPE) or by compound-specific toxicity were evaluated using MTS assays. The $\mathrm{EC}_{50}$ and $\mathrm{CC}_{50}$ were calculated as abovementioned.

\subsection{Viral infection of reconstituted human airway epithelium cells}

Human tracheal airway epithelium cells (HtAEC; catalogue no. EP01MD) and human small airway epithelium cells (HsAEC; catalogue no. EP21SA) from healthy donors were obtained from Epithelix (Geneva, Switzerland) in an air-liquid interphase set-up. After arrival, the insert was washed with pre-warmed 1x PBS (Gibco, catalogue no. 14190-094) and maintained in corresponding MucilAir medium (Epithelix, catalogue no. EP04MM) or SmallAir medium (Epithelix, catalogue no. EP64SA) at $37{ }^{\circ} \mathrm{C}$ and $5 \% \mathrm{CO}_{2}$ for at least 4 days before use. On the day of the experiment, the HAEC were first pre-treated with basal medium containing compounds at different concentrations for indicated hours, followed by exposing to $100 \mu \mathrm{L}$ of SARS-CoV-2 inoculum from apical side for $1.5 \mathrm{~h}$. Then the cultures were incubated at the indicated temperatures. The first apical wash with PBS was collected either right after the removal of viral inoculum (day 0 ) or $24 \mathrm{~h}$ later (day 1 postinfection (p. i.)). Every other day from day 0, subsequent apical washes were collected whereas compound-containing medium in the basolateral side of the HAEC culture was refreshed. Wash fluid was stored at $-80{ }^{\circ} \mathrm{C}$ for following experiments.

\subsection{RNA extraction and quantitative reverse transcription-PCR (RT- qPCR)}

Viral RNA in the apical wash was isolated using the Cells-to-cDNA ${ }^{\mathrm{TM}}$ II cell lysis buffer kit (Thermo Fisher Scientific, catalogue no. AM8723). Briefly, $5 \mu \mathrm{L}$ wash fluid was added to $50 \mu \mathrm{L}$ lysis buffer, incubated at room temperature (RT) for $10 \mathrm{~min}$ and then at $75^{\circ} \mathrm{C}$ for $15 \mathrm{~min} .150 \mu \mathrm{L}$ nuclease-free water was additionally added to the mixture prior to RTqPCR. In parallel, a ten-fold serial dilution of corresponding virus 
stock was extracted. The amount of viral RNA expressed as TCID50 equivalent per insert (TCID50e/insert) was quantified by RT-qPCR using iTaq universal probes one-step kit (Bio-Rad, catalogue no. 1725141), and a commercial mix of primers for $\mathrm{N}$ gene (forward primer $5^{\prime}$ GACCCCAAAATCAGCGAAAT- $3^{\prime}$, reverse primer $5^{\prime}$-TCTGGTTACTGCCAGTTGAATCTG- $3^{\prime}$ ) and probes (5'-FAM-ACCCCGCATTACGTTTGGTGGACC-BHQ1-3') manufactured at IDT Technologies (catalogue no. 10006606). The reaction (final volume: $20 \mu \mathrm{L}$ ) consisted of $10 \mu \mathrm{L}$ one-step reaction mix $2 \mathrm{X}, 0.5 \mu \mathrm{L}$ reverse transcriptase, $1.5 \mu \mathrm{L}$ of primers and probes mix, $4 \mu \mathrm{L}$ nuclease-free water, and $4 \mu \mathrm{L}$ viral RNA. The RT-qPCR was executed on a Lightcycler 96 thermocycler (Roche), starting at $50^{\circ} \mathrm{C}$ for $15 \mathrm{~min}$ and $95^{\circ} \mathrm{C}$ for $2 \mathrm{~min}$, followed by 45 cycles of $3 \mathrm{~s}$ at $95{ }^{\circ} \mathrm{C}$ and $30 \mathrm{~s}$ at $55^{\circ} \mathrm{C}$.

\subsection{Titration using a 50\% tissue culture infectious dose (TCID50) assay}

VeroE6 cells were seeded in 96-well tissue culture plates (Corning, catalogue no. 353072 ) at a density of $1 \times 10^{4}$ cells $/ 180 \mu \mathrm{L} /$ well. After $24 \mathrm{~h}$, serial 10-fold dilutions of ALI wash fluid were prepared in the plates. Cells were incubated for 3 days at $37{ }^{\circ} \mathrm{C}$ and evaluated microscopically for the absence or presence of virus induced cytopathic effect (CPE). The infectious viral titer was determined by end-point titration, expressed as TCID50/ml. Virus titers were calculated by using the Spearman and Karber method as previously reported (Kärber, 1931; Spearman).

\subsection{Statistical analysis}

All statistical comparisons in the study were performed in GraphPad Prism (GraphPad Software, Inc.). Statistical significance was determined using the ordinary one-way ANOVA with Dunnett's multiple comparison test. P-values of $\leq 0.05$ were considered statistically significant. In the figures "ns" indicates a $\mathrm{p}>0.05$. Asterisks indicate a statistical significance level of $* \mathrm{p}<0.05, * * \mathrm{p}<0.01,{ }^{* * *} \mathrm{p}<0.001, * * * * \mathrm{p}<$ 0.0001 .

\section{Results}

\subsection{Replication kinetics of SARS-CoV-2 in reconstituted human airway epithelia}

We first compared the replication kinetics of the Belgian isolate GHB03021 and the German isolate BavPat1 of SARS-CoV-2. The main differences in the genomes of these viruses is the D614G amino acid change in the spike-protein of BavPat1 and the deletion of several amino acids near the furin-cleavage site in the GHB-03021 isolate (because of extensive passaging in VeroE6 cells). The replication kinetics was investigated at respectively 35 and $37^{\circ} \mathrm{C}$ in both cultures from tracheal cells (HtAEC) or from small airway cells (HsAEC). In preliminary experiments, it was observed that an input of $10^{2}, 10^{3}$ or $10^{4}$ TCID50/ insert resulted in comparable levels of virus production (data not shown). We therefore selected $2 \times 10^{3}$ TCID50/insert as the viral input for this experiment. Overall, BavPat1 infected the cultures more efficiently than the GHB-03021 isolate did (Fig. 1). For example, at $37{ }^{\circ} \mathrm{C}$ not all bronchiole-airway derived inserts infected with GHB-03021 resulted in a productive infection whereas all cultures infected with BavPat1 showed productive infection under all conditions. Virus replication was for both isolates higher at $35^{\circ} \mathrm{C}$ and more reproducible as compared with a temperature of $37{ }^{\circ} \mathrm{C}$. This difference was more pronounced for the HsAEC than for the HtAEC cultures.

\subsection{Effect of selected antivirals on SARS-CoV-2 replication in HAEC cultures}

Four nucleoside analogues that are known inhibitors of SARS-CoV-2 replication were selected as reference for studies in HAEC cultures.
These included remdesivir, GS-441524 (Li et al., 2021; Pruijssers et al., 2020; Shi et al., 2021; Xie et al., 2020; Zandi et al., 2020) (the parent nucleoside of remdesivir), EIDD-1931 (Zandi et al., 2020; Good et al., 2021; Sheahan et al., 2020) (the parent nucleoside of molnupiravir) and AT-511 (Good et al., 2021) [a guanosine nucleotide analogue with activity against hepatitis $\mathrm{C}$ virus (HCV)]. To select a suitable concentration range of these molecules, we first explored their effect in VeroE6 and Huh7 cell lines. Treatment with remdesivir, GS-441524 and EIDD-1931 selectively inhibited SARS-CoV-2 GHB-03021 replication. On the other hand, AT-511 was entirely devoid of antiviral activity (Table 1). GS-441524 showed similar EC 50 values when tested in HAEC cultures in comparison with VeroE6 and Huh7 cells (Table 1). For remdesivir we observed $\sim 10$ x lower $\mathrm{EC}_{50}$ value in the HAEC culture and a $>100$ x lower $\mathrm{EC}_{50}$ value in the Huh7 cells when compared with the VeroE6 cells.

In a subsequent experiment, the activities of a concentration range of remdesivir and GS-441524 were tested on SARS-CoV-2 GHB-03021 in HtAEC cultures during 10 days of treatment (Fig. 2). Both compounds showed strong inhibition at $2 \mu \mathrm{M}$ throughout the experiment, suppressing virus replication below the limit of quantification. Remdesivir also showed inhibition at $0.4 \mu \mathrm{M}$ and at $0.08 \mu \mathrm{M}$ until day 4 . However, while remdesivir was still highly potent at $0.4 \mu \mathrm{M}\left(2 \log _{10}\right.$ reduction in vRNA), a steady increase in virus replication over time was observed at this concentration.

Next we explored the activity of GS-441524 in HtAEC cultures infected with SARS-CoV-2 GHB-03021 and in HsAEC cultures infected with BavPat1 (Fig. 3). At $10 \mu \mathrm{M}$, GS-441524 sterilized the HtAEC cultures from the GHB-03021 virus. Indeed, no virus production was detected during the first 9 days of treatment and, when treatment was stopped, no rebound was observed over the next 5 days of culturing. When evaluated at a concentration of $1 \mu \mathrm{M}$, GS-441524 reduced virus yield by $\sim 1 \log _{10}$ during the time of treatment, but lost activity once the compound was removed from the culture. In a separate experiment, GS441524 at $3 \mu \mathrm{M}$ resulted in complete inhibition of virus production upon infection with BavPat1 (Fig. 3F-H). Also, $10 \mu \mathrm{M}$ of EIDD-1931 resulted in a pronounced antiviral effect (Fig. 4F-J), whereas AT-511 was surprisingly devoid of any antiviral activity (Fig. 4A-E) at the different concentrations tested $(1$ and $10 \mu \mathrm{M})$.

\subsection{Prophylactic interferon type I and type III reduce SARS-CoV-2 production}

Human IFN has previously been used to treat several viral infections (Lokugamage et al., 2020; Strayer et al., 2014) and clinical trials against SARS-CoV-2 are ongoing (ClinicalTrials.gov number: NCT04315948, NCT04385095, and NCT04492475). We investigated whether IFN $\beta$-1a and IFN $\lambda 1$ exert antiviral activity when used as a prophylactic monotherapy. Tracheal cultures were pre-treated with either 5 and $50 \mathrm{ng} / \mathrm{mL}$ IFN $\lambda 1(5 \mathrm{ng} / \mathrm{mL}$ is the average concentration secreted in the basal medium of infected HAEC cultures (Essaidi-Laziosi et al., 2018)) or 1 and $100 \mathrm{IU} / \mathrm{mL}$ IFN $\beta-1 \mathrm{a}$ for $24 \mathrm{~h}$, and subsequently infected with BavPat1. Both drugs were able to reduce viral titers in a dose-dependent manner (Fig. 5A and F). Viral loads were reduced by $100 \mathrm{IU} / \mathrm{mL}$ IFN $\beta-1 \mathrm{a}\left(3.3 \log _{10}\right.$ vRNA reduction, $3.6 \log _{10}$ titer reduction) and $50 \mathrm{ng} / \mathrm{mL}$ IFN $\lambda 1$ ( $4.2 \log _{10}$ vRNA reduction, $5.0 \log _{10}$ titer reduction) on day 4 p. i. (Fig. 5B, D, 5G, 5I respectively). At later time points viral load in the treated samples increased again.

\section{Discussion}

We demonstrate that ex vivo models reconstituted from human tracheal or small airway epithelium are permissive for SARS-CoV-2 infection and robustly produces viral progenies from the apical side in long-term experiments (up to 14 days p. i.). Recent studies have also reported on the effect of different SARS-CoV-2 isolates and incubation temperatures on virus replication kinetics (Corman et al., 2016; Pohl et al., 2021; V'kovski et al., 2021; Zhu et al., 2021). We tested two 
Table 1

Antiviral activity against SARS-CoV-2 in cell lines and HAEC cultures.

\begin{tabular}{|c|c|c|c|c|c|c|c|}
\hline \multirow[t]{2}{*}{ Compounds } & \multicolumn{3}{|l|}{ VeroE6-GFP } & \multicolumn{3}{|l|}{ Huh7 } & \multirow{2}{*}{$\frac{\text { HAEC }}{\mathrm{EC}_{50}(\mu \mathrm{M})}$} \\
\hline & $\mathrm{EC}_{50}(\mu \mathrm{M})$ & $\mathrm{CC}_{50}(\mu \mathrm{M})$ & SI & $\mathrm{EC}_{50}(\mu \mathrm{M})$ & $\mathrm{CC}_{50}(\mu \mathrm{M})$ & SI & \\
\hline GS-441524 & $0.78-0.89$ (75) & 49-83 (49) & $>50$ & $1.1-1.5(9)$ & $37-59$ (11) & $>20$ & 0.51 \\
\hline AT-511 & $>100(4)$ & $>100(4)$ & ND & $>100(2)$ & $>100(2)$ & ND & $>10$ \\
\hline EIDD-1931 & $0.57-0.87(8)$ & $>100(8)$ & $>100$ & $1.3-1.4(2)$ & $11-12(2)$ & 8.5 & $<10$ \\
\hline Remdesivir & $0.74-1.34(8)$ & $>100(10)$ & $>100$ & $<0.001(6)$ & $0.3-2.2(6)$ & $>1000$ & 0.048 \\
\hline
\end{tabular}

Data on cell lines represents the interquartile range (Q1-Q3) and the number of independent measurements (n). Data on HAEC cultures are obtained from the experiments described in Figs. 2 and 4 (both at day 6 p.i.). Abbreviations: SI, selectivity index. ND: not determined.
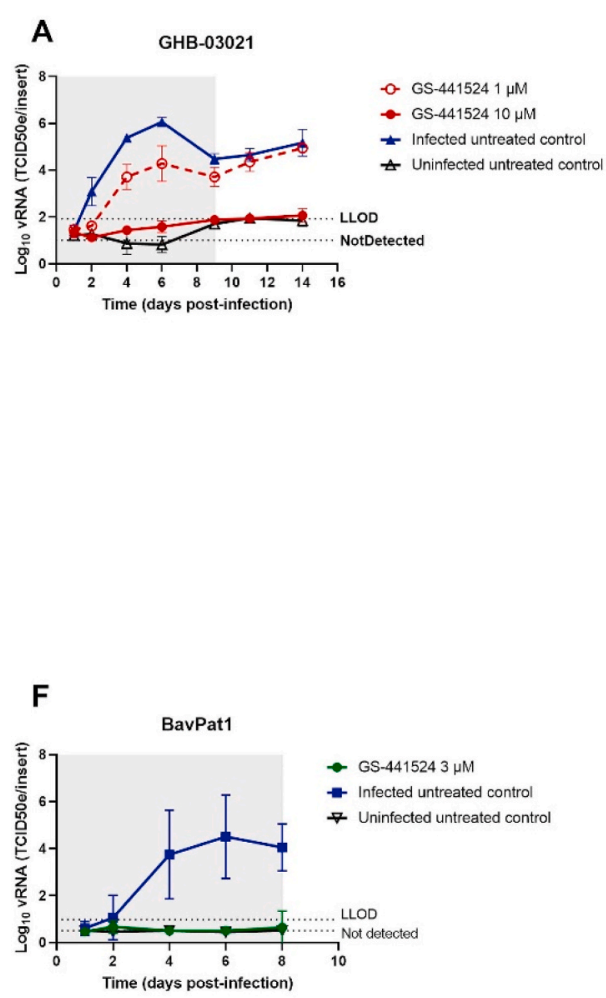
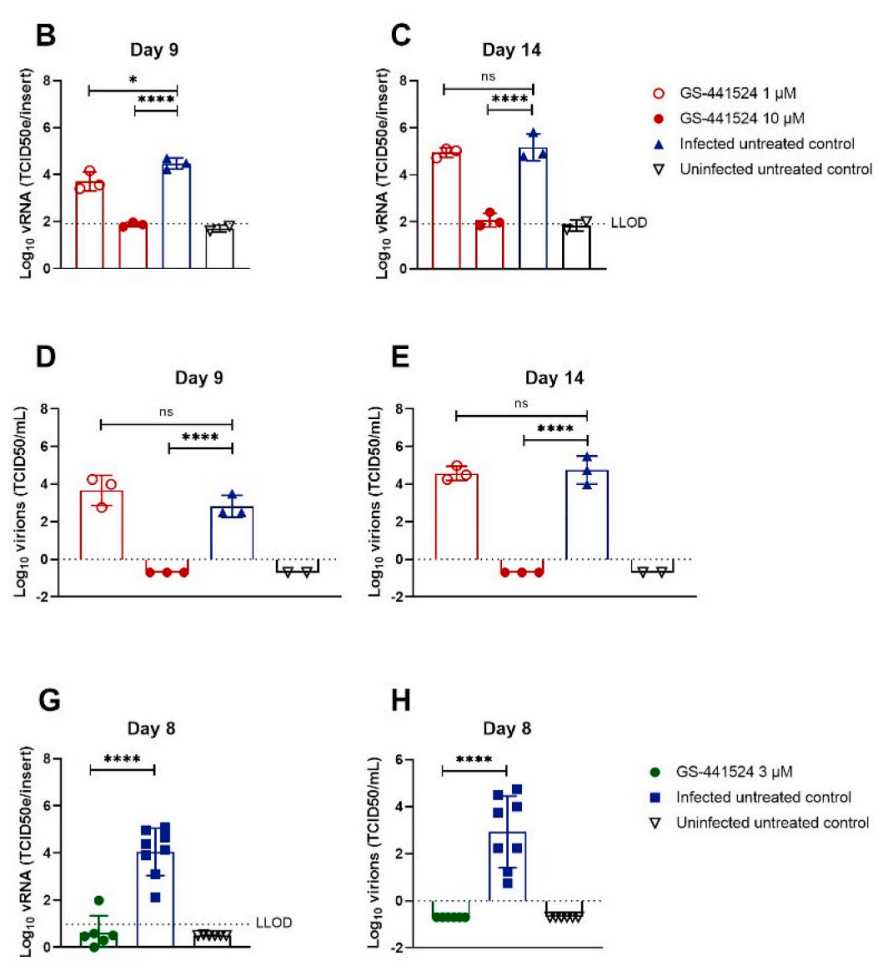

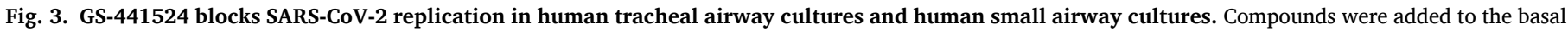

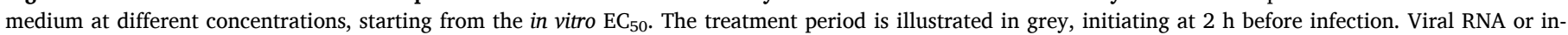

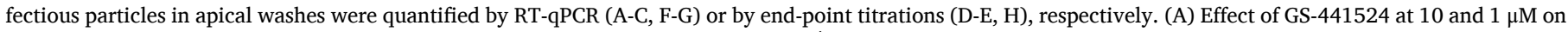

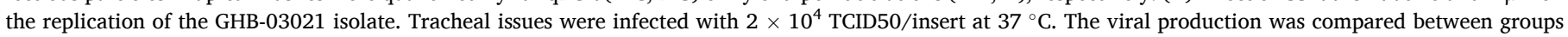

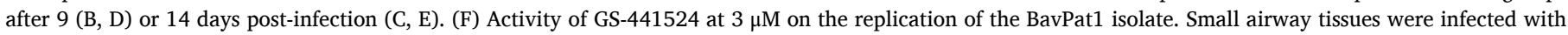

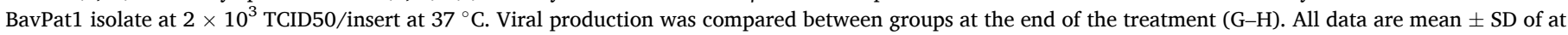

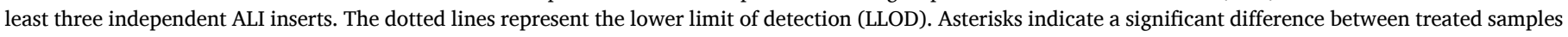
and infected untreated control. ${ }^{*} \mathrm{p}<0.05,{ }^{* *} \mathrm{p}<0.01,{ }^{* * *} \mathrm{p}<0.001, * * * * \mathrm{p}<0.0001$.

isolates, BavPat1 and GHB-03021, and found that BavPat1 proved to be more infectious. The BavPat1 isolate carries the p. D614G substitution in the spike (S) protein, which has been reported to increase the stability and infectivity of virions in HAEC culture by enhancing the ACE2-receptor-binding (Korber et al., 2020; Yurkovetskiy et al., 2020). Isolates with this p. D614G substitution have become globally dominant (Korber et al., 2020; Yurkovetskiy et al., 2020; Plante et al., 2020; Zhang et al., 2020). Whereas the GHB-03021 has a deletion of several amino-acids in the S1/S2 boundary that is typically found in VeroE6-adapted isolates (Boudewijns et al., 2020). Continued propagation of SARS-CoV-2 in Vero cells are reported to cause several substitutions and/or deletions in the S1/S2 boundary (Zhu et al., 2021; Davidson et al., 2020; Lau et al., 2020; Liu et al., 2020; Ogando et al., 2020), which are only rarely observed in clinical samples (Lau et al., 2020; Liu et al., 2020). We speculate that the adaptation to Vero cells results in a phenotype that allows more efficient entry through an ACE2-independent pathway. This entry mechanism would enhance entry in VeroE6 cells but would limit entry in primary lung epithelial cells. Further mechanistic studies are required to elucidate this hypothesis.

The anatomical distance and ambient temperature between upper and lower human respiratory tracts have a profound influence on the replication kinetics of respiratory viruses (Corman et al., 2016; Tyrrell and Bynoe, 1965; Holwerda et al., 2019; Kendall et al., 1962). In agreement with other studies, we observed SARS-CoV-2 growth in favour of lower temperature $\left(35^{\circ} \mathrm{C}\right)$ which can be attributed to the temperature preference of SARS-CoV-2 S protein for its folding and transport (V'kovski et al., 2021; Laporte et al., 2021). Both primary HtAEC and HsAEC cultures are shown to be a robust model for SARS-CoV-2 replication that can be used for antiviral drug profiling.

A promising target for the development of novel antiviral agents active against coronaviruses is the viral RNA-dependent RNA polymerase (RdRp) (teVelthuis, 2014). Remdesivir, a phosphoramidate prodrug of an adenosine C-nucleoside, was approved as the first COVID-19 

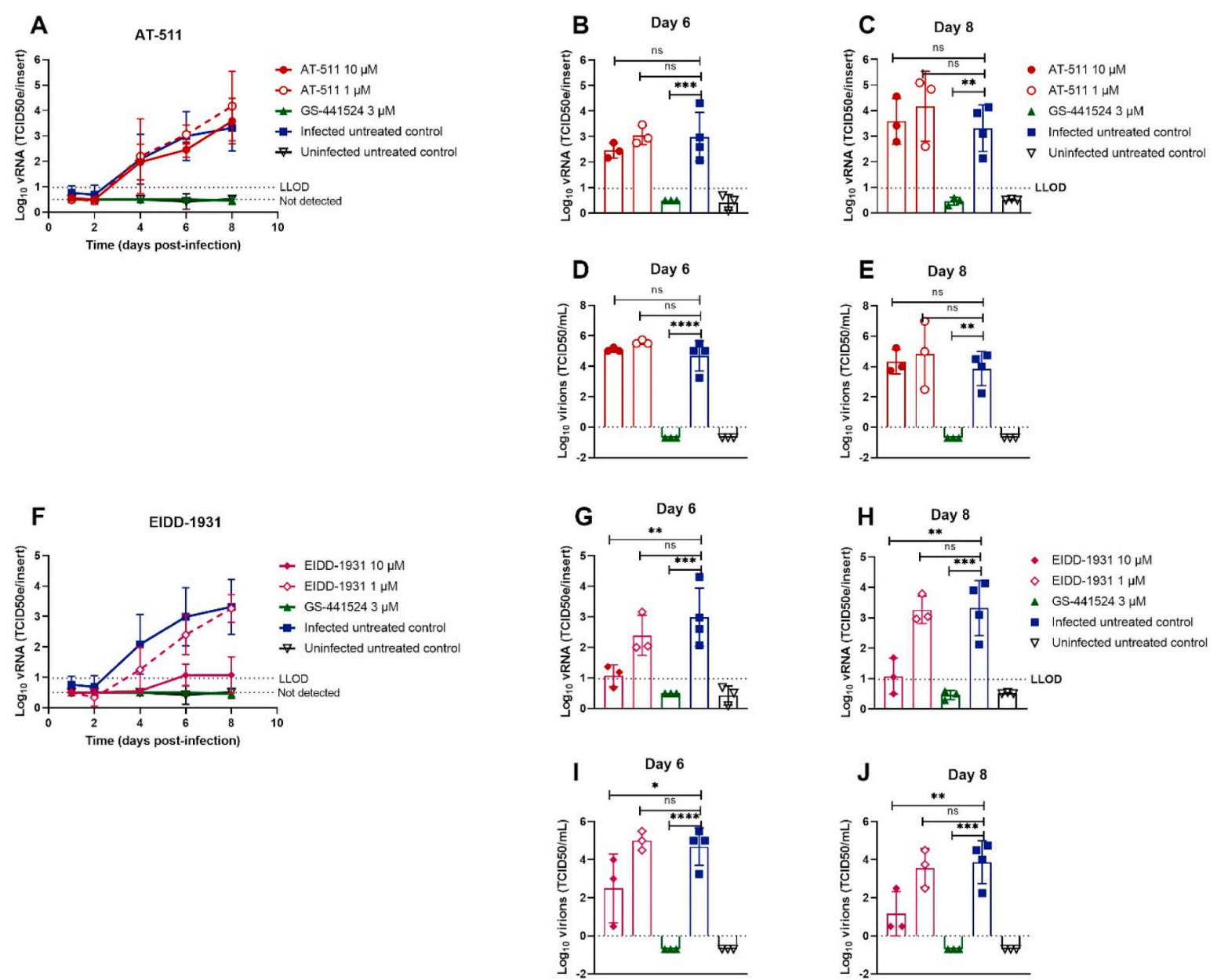

Fig. 4. Assessment of antiviral activity of AT-511 and EIDD-1931 against SARS-CoV-2 in primary human tracheal airway cultures. Compounds were added to the basal medium at different concentrations, starting $1 \mathrm{~h}$ before infection with BavPat 1 isolate at $2 \times 10^{3} \mathrm{TCID} 50 /$ insert at $37^{\circ} \mathrm{C}$. Basal medium, with or without compounds, was refreshed every other day from day 0 to day 8. Viral RNA or infectious particles in apical washes were quantified by RT-qPCR (A-C, F-H) or by endpoint titrations (D-E, I-J), respectively. Dose-response and time-dependent activity of AT-511 (A) and EIDD-1931 (F). Comparison of the viral production between groups after 6 (B, D, G, I) or 8 days post-infection (C, E, H, J). All data are mean \pm SD of at least three replicates. The dotted lines represent the lower limit of detection (LLOD). Asterisks indicate a significant difference between treated samples and infected untreated control. *p $<0.05,{ }^{* *} \mathrm{p}<0.01,{ }^{* * *} \mathrm{p}<0.001,{ }^{* * * *} \mathrm{p}$ $<0.0001$.

therapy for the treatment of hospitalized patients based on several randomized clinical studies. Our experiments showed that remdesivir had strong SARS-CoV-2 antiviral potency at $2 \mu \mathrm{M}$ and intermediate potency at $0.4 \mu \mathrm{M}$ for up to 10 days of treatment. Partial viral suppression persisted at $0.08 \mu \mathrm{M}$ until 4 days p. i. These results are in agreement with an earlier study (Pruijssers et al., 2020), where the virus replication was monitored for 3 days. We demonstrated that the parent nucleoside GS-441524 (Li et al., 2021; Pruijssers et al., 2020; Shi et al., 2021; Xie et al., 2020; Zandi et al., 2020) at $2 \mu \mathrm{M}$ can also "sterilize" HAEC cultures from SARS-CoV2 as no rebound of the virus was noted several days after removal of the molecule. At $1 \mu \mathrm{M}$ GS-441524, an intermediate antiviral potency was observed, but significant antiviral activity was not observed at $0.4 \mu \mathrm{M}$ in the HAEC cultures. Differences in antiviral potencies of remdesivir and GS-441524 have been reported depending on the cell lines used, which correlates with the formation of the biologically active ( $5^{\prime}$-triphosphate) metabolite (Li et al., 2021; Pruijssers et al., 2020). For remdesivir we observed $\sim 10 \mathrm{x}$ lower $\mathrm{EC}_{50}$ value in the HAEC culture and a $>100 x$ lower $\mathrm{EC}_{50}$ value in the Huh7 cells when compared with the VeroE6 cells. These results indicate that it is important to use the most relevant target cell type for the quantification of antiviral activity confirming that HAEC culture is the superior model when it comes to drug screening. The oral bioavailability of GS-441524, in addition, has been reported to be low in rats and monkey, but high in dog (Mackman et al., 2021), suggesting that oral delivery of GS-441524 in human is not easy to predict.

AT-527 is currently being evaluated in phase II clinical trials for COVID-19 (ClinicalTrials.gov). Anti-SARS-CoV-2 activity of AT-511, the free base form of AT-527, was not observed in VeroE6, Huh7 cells nor in the HAEC cultures. This is in contrast with a recent publication where sub-micromolar activities of AT-511 were observed in very similar assay systems (Good et al., 2021). Currently, there is no data to reconcile this discrepancy. One possibility is that small differences in the assay conditions may substantially influence the metabolism of AT-511 to its active form and consequently impact its antiviral activity. As AT-511 is a double prodrug requiring activation by multiple enzymatic processes, it may be more susceptible to differential experimental conditions. The implications of these observations for in vivo efficacy are not clear, but should be further investigated.

The effect of EIDD-1931, which is the parent nucleoside of the ester prodrug molnupiravir (EIDD-2801), was also investigated. EIDD-1931 has been reported to exert antiviral activity against various human coronaviruses and molnupiravir is currently in clinical trials for SARSCoV-2 (Good et al., 2021; Sheahan et al., 2020). Initial interim data from a phase II study provides first evidence for antiviral activity in COVID patients (https://www.croiconference.org/abstract/reductionin-infectious-sars-cov-2-in-treatment-study-of-covid-19-with-moln 

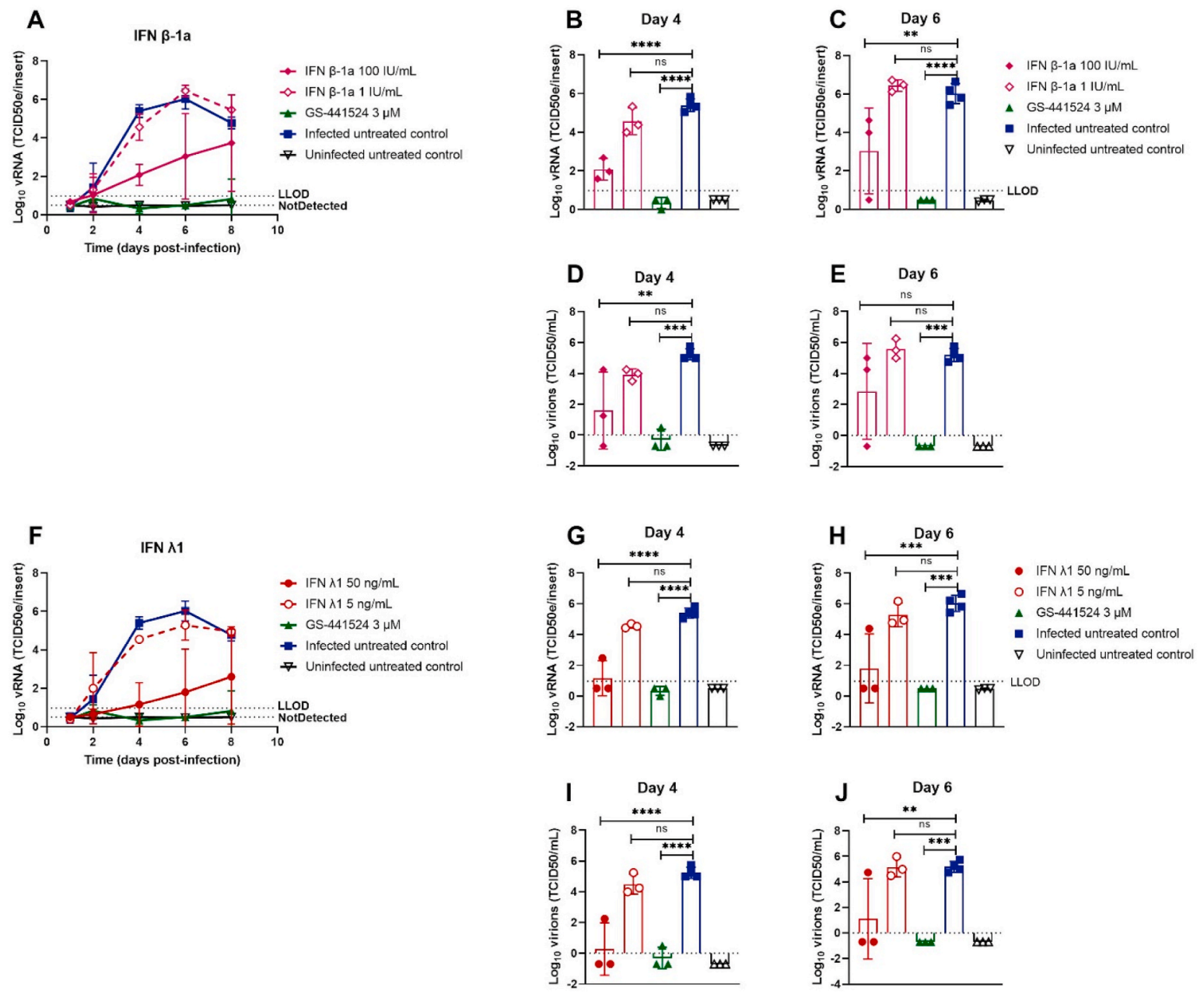

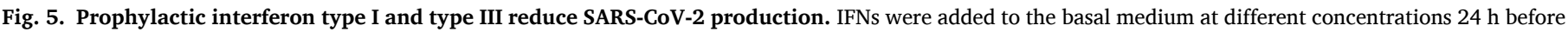

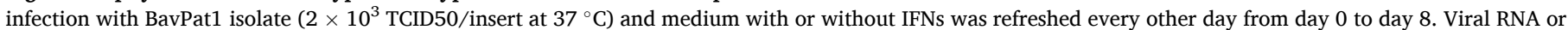

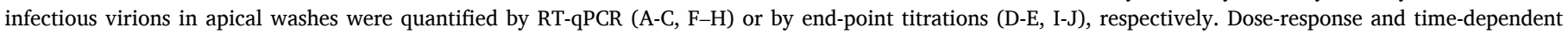

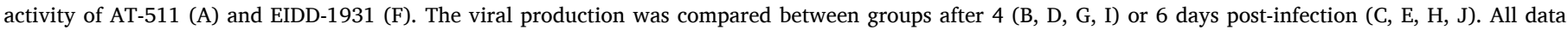

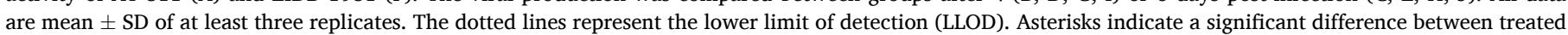
samples and infected untreated control. ${ }^{*} \mathrm{p}<0.05$, **p $<0.01, * * * \mathrm{p}<0.001,{ }^{* * * * \mathrm{p}}<0.0001$.

upiravir/). Treatment with EIDD-1931 resulted in a pronounced antiviral effect in HtAEC cultures at $10 \mu \mathrm{M}$, which was then lost at $1 \mu \mathrm{M}$. A previous study reported potent antiviral activity of EIDD-1931 against SARS-CoV-2 in HAEC at $0.1 \mu \mathrm{M}$ and at 2 days p. i. (Sheahan et al., 2020). It remains to be investigated whether the longer incubation time ( 6 and 8 days p. i.) reduces the activity of EIDD-1931 similarly to other nucleoside analogues tested.

A significant inhibitory effect of IFN $\beta$-1a and IFN $\lambda 1$ was also noted, at high concentrations and in particular during the first few days of treatment. At later time-points, viral replication increased in the treated cultures, suggesting that the virus can escape the initial effect of IFN. The effective concentration of IFN $\beta$-1a used in this study is comparable with a clinically achievable concentration and is in line with other reports (Lokugamage et al., 2020; Strayer et al., 2014; Vanderheiden et al., 2020).

In conclusion, both the replication of two SARS-CoV-2 isolates in HAEC cultures and the antiviral effect of representative and clinically relevant nucleoside inhibitors were assessed, providing a reference for this physiologically relevant model and its effective use in testing additional inhibitors of SARS-CoV2 replication.

\section{Author contributions}

J.N. and D.J. conceptualized and supervised the project. T.N.D.D, D. J., J.P.B., and T.C. designed the research. T.N.D.D. and K.D. performed the ALI-related experiments. T.N.D.D., L.V., and D.J. analysed data. A.J. C, P.A.G, and M.D.B. characterized AT-511 structure and tested its activity against HCV. T.N.D.D. wrote the first draft of the manuscript. D.J., S.D.J., L.V., and J.N. edited the manuscript. L.V., D.J. and J.N. acquired the funding.

\section{Declaration of competing interest}

The authors declare the following financial interests/personal relationships which may be considered as potential competing interests: $\mathrm{J}$. N. received a contract from Gilead to support part of the studies reported herein. These authors are employees of Gilead: J.P.B. and T.C.

\section{Acknowledgements}

We thank Birgit Voeten, Niels Cremers, Tina van Buyten and Thibault Francken for their excellent technical assistance. We also thank Piet Maes for kindly providing the SARS-CoV-2 GHB-03021/2020 isolate used in this study. This research was partially supported by Bill \& 
Melinda Gates Foundation (BMGF) under grant number INV-006366, the Covid-19-Fund KU Leuven/Universitair Ziekenhuis Leuven and the COVID-19 call of Fund for Scientific Research Flanders (FWO) (grant G0G4820N), the European Union's Horizon 2020 research and innovation program under Grant Agreement 101003627 (Swift COronavirus therapeutics REsponse project). T.N.D.D received the fellowship from European Union's Horizon 2020 research and innovation programme under Marie Sklodowska-Curie grant agreement No. 812673 (OrganoVIR project). Part of this research work was performed using the 'CapsIt' research infrastructure (project ZW13-02) that was financially supported by the Hercules Foundation and Rega Foundation, KU Leuven.

\section{References}

Beigel, J.H., Tomashek, K.M., Dodd, L.E., et al., 2020. Remdesivir for the treatment of covid-19 - final report. N. Engl. J. Med. 383 (19), 1813-1826.

Boda, B., Benaoudia, S., Huang, S., et al., 2018. Antiviral drug screening by assessing epithelial functions and innate immune responses in human 3D airway epithelium model. Antivir. Res. 156, 72-79.

Bohmer, M.M., Buchholz, U., Corman, V.M., et al., 2020. Investigation of a COVID-19 outbreak in Germany resulting from a single travel-associated primary case: a case series. Lancet Infect. Dis. 20 (8), 920-928.

Boudewijns, R., Thibaut, H.J., Kaptein, S.J.F., et al., 2020. STAT2 signaling restricts viral dissemination but drives severe pneumonia in SARS-CoV-2 infected hamsters. Nat. Commun. 11 (1), 5838.

Corman, V.M., Eckerle, I., Memish, Z.A., et al., 2016. Link of a ubiquitous human coronavirus to dromedary camels. Proc. Natl. Acad. Sci. U. S. A. 113 (35), 9864-9869.

Davidson, A.D., Williamson, M.K., Lewis, S., et al., 2020. Characterisation of the transcriptome and proteome of SARS-CoV-2 reveals a cell passage induced in-frame deletion of the furin-like cleavage site from the spike glycoprotein. Genome Med. 12 (1), 68.

Dellicour, S., Durkin, K., Hong, S.L., et al., 2021. A phylodynamic workflow to rapidly gain insights into the dispersal history and dynamics of SARS-CoV-2 lineages. Mol. Biol. Evol. 38 (4), 1608-1613.

Essaidi-Laziosi, M., Brito, F., Benaoudia, S., et al., 2018. Propagation of respiratory viruses in human airway epithelia reveals persistent virus-specific signatures. J. Allergy Clin. Immunol. 141 (6), 2074-2084.

Good, S.S., Westover, J., Jung, K.H., et al, 2021. AT-527, a double prodrug of a guanosine nucleotide analog, is a potent inhibitor of SARS-CoV-2 in vitro and a promising oral antiviral for treatment of COVID-19. Antimicrob. Agents Chemother. 65 (4), e02479-20.

Holwerda, M., Kelly, J., Laloli, L., et al., 2019. Determining the replication kinetics and cellular tropism of influenza D virus on primary well-differentiated human airway epithelial cells. Viruses 11 (4), 377.

Jochmans, D., Leyssen, P., Neyts, J., 2012. A novel method for high-throughput screening to quantify antiviral activity against viruses that induce limited CPE. J. Virol. Methods 183 (2), 176-179.

Kärber, G., 1931. Beitrag zur kollektiven Behandlung pharmakologischer Reihenversuche. Schmiedeb. Arch. für Exp. Pathol. Pharmakol. 162 (4), 480-483.

Kendall, E.J., Bynoe, M.L., Tyrrell, D.A., 1962. Virus isolations from common colds occurring in a residential school. Br. Med. J. 2 (5297), 82-86.

Korber, B., Fischer, W.M., Gnanakaran, S., et al., 2020. Tracking changes in SARS-CoV-2 spike: evidence that D614G increases infectivity of the COVID-19 virus. Cell 182 (4), 812-827 e819.

Laporte, M., Raeymaekers, V., Van Berwaer, R., et al., 2021. The SARS-CoV-2 and other human coronavirus spike proteins are fine-tuned towards temperature and proteases of the human airways. PLoS Pathog. 17 (4) e1009500.
Lau, S.Y., Wang, P., Mok, B.W., et al., 2020. Attenuated SARS-CoV-2 variants with deletions at the S1/S2 junction. Emerg. Microb. Infect. 9 (1), 837-842.

Li, Y., Cao, L., Li, G., et al., 2021. Remdesivir metabolite GS-441524 effectively inhibits SARS-CoV-2 infection in mouse models. J. Med. Chem.

Liu, Z., Zheng, H., Lin, H., et al., 2020. Identification of common deletions in the spike protein of severe acute respiratory syndrome coronavirus 2. J. Virol. 94 (17),

Lokugamage, K.G., Hage, A., de Vries, M., et al., 2020. Type I interferon susceptibility distinguishes SARS-CoV-2 from SARS-CoV. J. Virol. 94 (23).

Loo, S.L., Wark, P.A.B., Esneau, C., Nichol, K.S., Hsu, A.C., Bartlett, N.W., 2020. Human coronaviruses $229 \mathrm{E}$ and OC43 replicate and induce distinct antiviral responses in differentiated primary human bronchial epithelial cells. Am. J. Physiol. Lung Cell Mol. Physiol. 319 (6), L926-L931.

Mackman, R.L., Hui, H.C., Perron, M., et al., 2021. Prodrugs of a 1'-CN-4-Aza-7,9dideazaadenosine C-nucleoside leading to the discovery of remdesivir (GS-5734) as a potent inhibitor of respiratory syncytial virus with efficacy in the african green monkey model of RSV. J. Med. Chem. 64 (8), 5001-5017.

Murgolo, N., Therien, A.G., Howell, B., et al., 2021. SARS-CoV-2 tropism, entry, replication, and propagation: considerations for drug discovery and development. PLoS Pathog. 17 (2), e1009225.

Ogando, N.S., Dalebout, T.J., Zevenhoven-Dobbe, J.C., et al., 2020. SARS-coronavirus-2 replication in Vero E6 cells: replication kinetics, rapid adaptation and cytopathology. J. Gen. Virol. 101 (9), 925-940.

Plante, J.A., Liu, Y., Liu, J., et al., 2020. Spike mutation D614G alters SARS-CoV-2 fitness. Nature 592 (7852), 116-121.

Pohl, M.O., Busnadiego, I., Kufner, V., et al., 2021. SARS-CoV-2 variants reveal features critical for replication in primary human cells. PLoS Biol. 19 (3) e3001006.

Pruijssers, A.J., George, A.S., Schafer, A., et al., 2020. Remdesivir inhibits SARS-CoV-2 in human lung cells and chimeric SARS-CoV expressing the SARS-CoV-2 RNA polymerase in mice. Cell Rep. 32 (3), 107940.

Rut, W., Groborz, K., Zhang, L., et al., 2021. SARS-CoV-2 M(pro) inhibitors and activitybased probes for patient-sample imaging. Nat. Chem. Biol. 17 (2), 222-228.

Sheahan, T.P., Sims, A.C., Zhou, S., et al., 2020. An orally bioavailable broad-spectrum antiviral inhibits SARS-CoV-2 in human airway epithelial cell cultures and multiple coronaviruses in mice. Sci. Transl. Med. 12 (541).

Zhu, Y., Feng, F., Hu, G., et al., 2021. A genome-wide CRISPR screen identifies host factors that regulate SARS-CoV-2 entry. Nat Commun. 12 (1), 961.

Spearman C. The method of 'right and wrong cases' ('constant stimuli') without Gauss's formulae. Br. J. Psychol..2:227-242.

Shi, Y., Shuai, L., Wen, Z., et al., 2021. The preclinical inhibitor GS441524 in combination with GC376 efficaciously inhibited the proliferation of SARS-CoV-2 in the mouse respiratory tract. Emerg Microbes Infect. 10 (1), 481-492.

Strayer, D.R., Dickey, R., Carter, W.A., 2014. Sensitivity of SARS/MERS CoV to interferons and other drugs based on achievable serum concentrations in humans. Infect. Disord. - Drug Targets 14 (1), 37-43.

te Velthuis, A.J., 2014. Common and unique features of viral RNA-dependent polymerases. Cell. Mol. Life Sci. 71 (22), 4403-4420.

Tyrrell, D.A., Bynoe, M.L., 1965. Cultivation of a novel type of common-cold virus in organ cultures. Br. Med. J. 1 (5448), 1467-1470.

Vanderheiden, A., Ralfs, P., Chirkova, T., et al., 2020. Type I and type III interferons restrict SARS-CoV-2 infection of human airway epithelial cultures. J. Virol. 94 (19).

V'kovski, P., Gultom, M., Kelly, J., et al., 2021. Disparate temperature-dependent virus host dynamics for SARS-CoV-2 and SARS-CoV in the human respiratory epithelium. PLoS Biol. 19 (3) e3001158.

Xie, X., Muruato, A.E., Zhang, X., et al., 2020. A nanoluciferase SARS-CoV-2 for rapid neutralization testing and screening of anti-infective drugs for COVID-19. Nat. Commun. 11 (1), 5214.

Yurkovetskiy, L., Wang, X., Pascal, K.E., et al., 2020. Structural and functional analysis of the D614G SARS-CoV-2 spike protein variant. Cell 183 (3), 739-751 e738.

Zandi, K., Amblard, F., Musall, K., et al., 2020. Repurposing nucleoside analogs for human coronaviruses. Antimicrob. Agents Chemother. 65 (1).

Zhang, L., Jackson, C.B., Mou, H., et al., 2020. SARS-CoV-2 spike-protein D614G mutation increases virion spike density and infectivity. Nat Commun., 6013 